Gefässchirurgie 2009 · 14:8-8

DOI 10.1007/s00772-008-0662-0

๑) Springer Medizin Verlag 2009

\author{
T. Schmitz-Rixen \\ Klinikum der Goethe-Universität, Frankfurt am Main
}

\title{
Forschung als Grundlage der Gefäßchirurgie
}

Die in diesem Heft von drei verschiedenen Arbeitsgruppen vorgestellten Modelle experimenteller Forschung zeigen exemplarisch eindrücklich, dass das in unsere Gesellschaft für Gefäß- und Endovasculäre Chirurgie getragene Signal, sich um die vasculäre Biologie oder auch formuliert um die Grundlagen unseres Faches zu kümmern, auf fruchtbaren Boden gefallen ist. Diese Beiträge wie auch andere auf dem letzten Jahreskongress vorgetragene Grundlagenforschungsarbeiten sind international konkurrenzfähig; auch deshalb weil sie teilweise in internationalen Forschungsnetzwerken integriert sind. Keine Frage, dass hiermit auch die Perspektive verbunden ist, europäische Forschungsgelder einzuwerben. Bemerkenswert ist auch, dass die Seniorautoren praktizierende Gefäßchirurgen sind und durch ihr Engagement in die Lage versetzt werden, die in vielen Forschungsbereichen beobachtete große Lücke zwischen Grundlagenforschung und klinischer Forschung zu füllen. Der Kliniker wird hier zum produktiven Katalysator einer effizienten Translation, wie sie von Grundlagenforschern in zunehmendem Maße gefordert wird.

In den hier vorgestellten Forschungsprogrammen ist erfreulicherweise auch der gefäßchirurgische Nachwuchs engagiert. Es wird für uns Ältere eine wichtige Aufgabe sein, die bereits Engagierten maximal zu fördern und weitere Mitarbeiter einzuschleusen. Die akademische Perspektive, die man hierdurch dem Nachwuchs aufzeigen kann, wird aus meiner Erfahrung wieder gerne angenommen. Wir müssen die Herausforderung annehmen, unsere hochmotivierten jungen
Mitarbeiter nicht nur durch ein klinisches sondern auch ein Forschungs-Curriculum in einem vertretbaren Zeitrahmen zu führen. Der kürzlich gegründete Konvent der universitären Gefäßchirurgen hat hierzu ein viel beachtetes Papier initiiert. Die vorgestellten Forschungsthemen stehen neben den endovasculären Verfahren im Zentrum der Zukunftsthemen unserer Fachgesellschaft. Auch bei den endovasculären Verfahren stände uns eine Investition in mehr Grundlagenforschung gut zu Gesicht. Die im Gesetzgebungsprozess stehenden Änderungen des Medizinproduktegesetzes in Richtung Arzneimittelgesetz könnten uns hier hinführen.

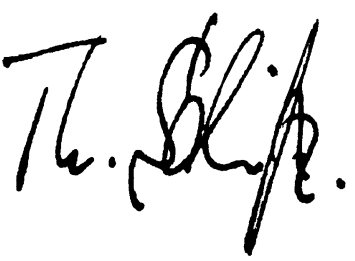

Univ.-Prof. Dr. Thomas Schmitz-Rixen

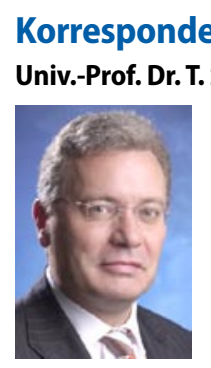

Univ.-Prof. Dr. T. Schmitz-Rixen

Klinik für Gefäß- und Endovascular-Chirurgie Klinikum der Goethe-Universität Research Associate: Max-Planck-Institute for Heart and Lung Research, Bad Nauheim Theodor-Stern-Kai-7, 60590 Frankfurt am Main schmitz-rixen@em.unifrankfurt.de 